\title{
LAS CONTRADICCIONES EN EL TRABAJO SOCIAL: UNA CRÍTICA AL EJERCICIO PROFESIONAL
}

\author{
Por: Carlos Andrés Zapata Calle
}

\begin{abstract}
"Nunca debemos sentirnos satisfechos con nuestros éxitos. Debemos refrenar la autosatisfacción y criticar constantemente nuestros defectos al igual que nos lavamos la cara y barremos el suelo diariamente para quitar el polvo y mantenerlos limpios".
\end{abstract}

Mao Tse Tung (1893-1976) Máximo dirigente de la República de China.

Hablar de trabajo social podría convertirse en una de las hazañas más complicadas de cumplir, pues, verdaderamente es una disciplina compleja, diversa y llena de matices que en ocasiones las palabras se quedan cortas para describirla. Es que para hablar de trabajo social, debemos hablar de realidades, de contextos, de sujetos, de problemáticas, de perspectivas, de conceptos; en fin, son tantos y tantos temas que componen nuestra profesión, que fácilmente llenamos páginas completas intentando explicar alguno de estos tópicos.

Pero si trabajo social es una profesión tan amplia, podríamos suponer que tiene un gran papel dentro de la sociedad, que el accionar de cada uno de sus profesionales es determinante en las dinámicas suscitadas al interior de cada comunidad -debería ser así-. Pero la realidad dice todo lo contrario. Podemos escoger una tarde y salir a la calle para preguntarle a ciudadanos y ciudadanas de a pie qué creen ellos y ellas qué es trabajo social, ¿Cuál será su respuesta? Sencillamente algunos responderán que no tienen ni idea, otros, que es algo así como ayudar, que es una profesión que estudian las mujeres, e incluso hasta podrían responder, que es el arte de entregar mercados y hacer encuestas. Una carrera compuesta de tan riquísimos elementos tiene tan pobre visión en el seno social... ique irónico! Pues, se supone que trabajo social se enfoca en las realidades sociales, en los sujetos y estos mismos sujetos son los que no validan de ninguna manera lo que la carrera hace. ¿Por qué ocurrirá esto? A otras profesiones no les pasa: no sucede con derecho, con medicina, ni incluso con psicología, pero sí con trabajo social.

Este asunto me ha cuestionado desde el primer momento que inicié a estudiar esta carrera, la cual amo y disfruto. Pero me inquieta profundamente, que muchos colegas que pasaron por las mismas aulas que yo pasé, que 
escucharon el discurso de empoderamiento, de exigencia de derechos, de transformación social, hoy en día cuando se encuentran del otro lado del río se enfrentan con una realidad que no tiene nada que ver con lo visto en los salones, y tal es el choque y la decepción, que muchos de ellos preferirían no haber tomado el camino de trabajo social.

Cuando me cuestiono por algo, me ocupo de analizarlo, y por supuesto que mi profesión no iba a ser la excepción, por lo que en clases estoy atento de cada palabra que mis docentes dicen, busco la oportunidad para compartir ideas con compañeros de otros semestres y en la medida de las posibilidades, entablo tertulias con chicos y chicas que se encuentran en prácticas o que ya están en el campo laboral; todo con el fin de entender que es lo que sucede al interior de trabajo social.

Lo primero que encontré, es que la gran mayoría de las personas que inician en trabajo social, simplemente no habían contemplado esta carrera como una posibilidad en su vida, es decir, llegaron aquí por accidente, porque querían psicología, porque era la única manera de pasar en la universidad, porque le estaban huyendo a las matemáticas, entre muchas otras razones. Entonces, qué se puede esperar de una carrera, en la que sus profesionales ingresan por el desconocimiento o por imposición de las circunstancias. Es obvio que una vez que salen de la universidad se sienten frustrados y no la ejercen con el amor, compromiso y determinación que se requiere, reproduciendo muchos de los estereotipos que estigmatizan nuestra profesión.

\section{Trabajo social, una profesión ecléctica}

or otro lado, trabajo social cuenta con un elemento que en muchos casos las y los estudiantes no lo tienen tan claro, y es el hecho que

_ nuestra carrera es una profesión ecléctica. Algunos ni siquiera saben el significado de esta expresión, otros no tienen ni idea que tiene que ver esto con trabajo social. Como sabemos, el trabajo social se nutre de todas las ciencias y disciplinas de las ciencias sociales, para contar con una visión holística de las realidades. Esto le permite efectuar lecturas aterrizadas a los contextos y así dar respuestas que realmente se orienten a la transformación de las problemáticas que se desarrollan en el seno de los grupos y poblaciones. Es por esta razón que decimos que trabajo social es ecléctico, porque la transdisciplinariedad y la multidisciplinariedad hacen parte de su fundamento epistemológico. El problema de este asunto, es que para entender lo que antes he explicado, se debe transitar por varios semestres, estrellarte una y otra vez con tus docentes por no emitir conceptos adecuados de la profesión y vivir un mar de 
confusiones, porque no sabes para que sirve lo que estás estudiando. Dentro del pénsum de trabajo social, encontramos asignaturas como: sociología, antropología, economía, pedagogía, entre muchas más; el estudiante cuando se enfrenta a esta variedad de conocimiento, en un principio no entiende que los aprendizajes que adquiere en estos cursos le serán fundamentales en su vida laboral, puesto que como lo plantea Edgar Morin (1994) ${ }^{1}$, las realidades son complejas y es en las realidades en que se desenvuelve el profesional de trabajo social. Por lo tanto es importante contar con los conocimientos necesarios para hacer un abordaje profesional a las situaciones o problemáticas con las que se está trabajando. Si esto se le dijera al estudiante de primer semestre, se le ahorrarían noches enteras de sufrimiento y podría contribuir a que los estudiantes se comprometan con su proceso académico, puesto que desde el primer momento comprenden la ruta que están siguiendo y el sentido de cada elemento que están asumiendo en la academia.

\section{Dilemas y contradicciones en el trabajo social}

Yontinuando con los aspectos que me parecen preocupantes de mi querida profesión, ahora quiero referirme a los constantes dilemas y contradicciones que se viven en ella. Una de las disputas más álgidas a las que me he enfrentado en mi tiempo de estudiante, es el asunto de qué método o perspectiva es mejor para acercarnos a una realidad -el famoso dilema de los métodos-. Muchos se ubican desde una visión cuantitativa de la realidad. Afirman que la única verdad es aquella que se puede "palpar y verificar", de lo contrario lo que se haga es considerado como meras opiniones que no están sustentadas científicamente, y por ello no se pueden tomar en cuenta como estudios que le aporten al conocimiento. Otros por su parte, satanizan todo lo relacionado con el positivismo, pues sostienen que este paradigma es tradicional y que no tienen en cuenta las particularidades de los individuos, sino que los mete a todos en un mismo saco, perdiendo de vista elementos fundamentales que pueden ser determinantes a la hora de hacer investigación o de intentar responder a una problemática. La propuesta en este sentido es el enfoque cualitativo y sus diversos métodos como la etnográfica, la investigación acción participativa, el interaccionismo simbólico entre muchos otros. Todos intentan rescatar los detalles y ubican a las y los sujetos en una posición de iguales, en donde todos, tanto profesional como población, pueden aportar conocimiento que ayude a aproximaciones más cercanas a la realidad. Estas dos maneras de entender la ciencia han estado paralelas, o en muchos casos enfrentadas, intentando mostrar cuál es la más adecuada para trabajar en ciencias sociales. Es una discusión interminable, que a mi modo de ver, impide una integralidad a la hora de producir conocimiento, pues, sea 
que se mire desde lo cuantitativo o lo cualitativo, ambas formas por si solas se ven permeadas por sesgos, que se sustentas en tontos egos ideológicos.

La doctora Elsa Bonilla, intenta superar esta pugna en su libro Más allá del dilema de los métodos (1997), y en su introducción nos propone algo muy interesante:

"El debate sobre el método más apropiado para estudiar la realidad social ha ocupado la atención de diversos pensadores e investigadores y ha devenido en una permanente inquietud en el desarrollo de las ciencias sociales desde sus mismos inicios. En los últimos diez años, y ante las restricciones evidentes y las crisis derivadas de un abordaje que da prioridad a lo mensurable 'por sobre todos los hechos'; la aproximación cualitativa rigurosa es cada vez más demandada y cuenta con crecientes adeptos, incluso en instancias de 'cuantificadores puros'. Estos pasos son tímidos todavía y se dan insistiendo en una visión un tanto peyorativa de la realidad subjetiva. Los evidentes vacíos de conocimiento, que han llevado, por un lado, a conclusiones científicas cuestionadas por las tendencias empíricas reales $y$, por otro, a la proyección y planeación de futuros inciertos y perversos para nuestras sociedades, están terminando por imponer la búsqueda de nuevos caminos. Aunque dichos caminos se nutren de las corrientes convencionales, sus restricciones pueden ser controladas y posiblemente superadas con otras propuestas, si se reconoce explícitamente que la realidad es objetiva-subjetiva y por lo tanto, los métodos de conocimiento deben ser suficientemente sensibles para captarla de manera total y no arbitrariamente escindida entre lo cuantificable y lo no cuantificable".

Este es un planteamiento fascinante, pues con unas simples pinceladas, la autora presenta el histórico debate dado en las ciencias sociales entre lo cuantitativo y lo cualitativo y sostiene que se deben encontrar nuevas formas que nos permitan conocer la realidad de una manera mucho más holística. No podemos -según ella- continuar fragmentando el conocimiento cuando este tiene aristas subjetivas y objetivas, y que la única manera de producir verdaderos saberes, es en la integralidad a la hora de abordar estar realidades. Entonces, esa discusión que a veces se vuelve demagógica y hasta sirve para sustentar la prepotencia intelectual de muchos profesionales, queda relegada al espacio del olvido para poder encontrar formas innovadoras que nos aproximen a los contextos estudiados.

Pero el tema de los métodos no es lo único por lo que se pelea en la carrera. Aún existen otros asuntos que tienen al trabajo social como la Patria Boba: 
discutiendo y discutiendo sin llegar a acuerdo, y el Pablo Morillo de la falta de identidad profesional, aborda nuestras costas para conquistarnos una vez más. Un asunto que a los ojos de muchos puede parecer tan simple, como la manera en que se denomina la acción de una profesión en su entorno, aquí es motivo de discusión: ¿intervenimos o no? Es la pregunta que muchos, a pesar de estar culminando su proceso académico aún se hacen, y lo peor es que hay colegas que ya están ejerciendo como trabajadores sociales, que no tiene claro cómo se podría llamar lo que ellos y ellas están desarrollando en sus campos laborales. La posibilidad de llegar a acuerdos en relación a este tópico -desde mi visión- es fundamental, pues, como lo anoté antes, el trabajo social carece de un posicionamiento dentro de la sociedad. Tal estatus lo considero necesario, entendiendo por un lado la importancia que tiene esta profesión en el abordaje de las realidades modernas, y por otro lado, la resistencia que existen de ciertos dirigentes de organizaciones o empresas de contratar trabajadores sociales, por el hecho que consideran que estos profesionales no hacen nada y que en vez de generar productividad lo que harán es acarrear gastos. Pero cómo no queremos que esta situación se dé, si al interior de nuestra propia carrera no podemos definir nuestro deber ser; y aún peor no podemos darle un nombre a nuestra labor profesional. En otras palabras, ¿Cómo queremos que los demás nos reconozcan, si no somos capases de reconocernos mostros mismos? Lo que afirmo, es respaldado en el documento Intervención social por Silvia Galeana de la O. y Nelia Tello Peón cuando afirman,

"En la literatura de Trabajo Social, la intervención social se ha definido dependiendo de la posición epistemológica y teórica que se adopte; así la ausencia y poca claridad en sus procesos de construcción de conocimiento, la debilidad de sus estructuras y soportes teóricos y el constante cuestionamiento respecto de su práctica (presencia del fantasma del empirismo, su utilidad, consistencia y cobertura de objetivos, coherencia en las estructura operativas de sus abordajes) provoca que estas diferencias en sus definiciones limiten producir una base de conocimiento sólida en torno a la intervención de Trabajo Social desde su muy particular perspectiva, que le permita aportar marcos explicativos y formas de actuación en las diversas manifestaciones y expresiones de la problemática que le plantea lo social".

Aquí es evidente, según estas autoras, que estas discusiones sin fin que vemos en las aulas de clase, no aportan al desarrollo de la profesión, sino todo lo contrario, sirven únicamente para generar ambigüedades e impedir que el trabajo social avance en su objetivo de generar nuevas maneras de entender y abordar lo social. 


\section{Entre las palabras y las acciones}

$\gamma$ ontinuando con este apasionante viaje por las distintas aristas del trabajo social, quiero referirme ahora al discurso que como profesionales empleamos en nuestra carrera. Hace unos días conversaba con una profesora del programa y le manifestaba mi preocupación sobre el tema de la deficiencia que tienen los y las estudiantes en la expresión oral. Ella de manera muy acertada, me respondió afirmando que las y los trabajadores sociales nos ganamos la vida hablando y escribiendo. Aquí podemos notar lo importante que es la palabra para nosotros. Se constituye como la herramienta con la cual contamos para llevar a cabo nuestra labor. Lo que me interesa anotar en este punto, es que en muchos casos he podido ver una incongruencia en muchos de mis compañeros y docentes: uno les escucha disertar en el aula de clase y manejan tan bien temas como el de empoderamiento, la justicia, la igualdad; y sencillamente es fascinante. Se sale del aula como con ganas de comerse el mundo, de salir a las calles y protestar, o de contarle a todos los que te encuentras en el camino lo que acabaste de aprender. Pero una vez tu comienzas a observar con detenimiento la vida y la cotidianidad de la persona que ha hablado, te puedes comenzar a cuestionar si verdaderamente eso que ha dicho está pasando por su ser o si simplemente son palabras ornamentarias que embellecen una clase o que sirven para obtener una nota. Lo que he escrito antes yo lo denomino hipocresía intelectual. Personas cargadas de ideologías fantásticas pero que a la hora de materialízalas pareciese que existiera una gran barrera entre el decir y el hacer. Son estos los colegas que mientras estaban en la academia, hablaban de forma elocuente, "con fundamento teórico, epistemológico, metodológico" y todas las arandelas que se le quería agregar. Pero una vez sale al campo de trabajo, todo el recorrido académico se echa a un lado, como si estuviese sufriendo de amnesia intelectual; y ya no habla de empoderamiento, ni de género, ni de nada, sino que se encarga de perpetuar el papel que de manera equívoca el trabajo social ha desempeñado por mucho tiempo en la sociedad.

En el trabajo social y en la vida misma se debe ser coherentes, de ir en línea con la filosofía que mis acciones den cuenta de mis palabras. Esto me da credenciales a la hora de hacer gestión, me permite tener una imagen transparente ante los que están a mi alrededor y me brinda cierta autoridad ética para cuestiona y criticar procesos mal llevados y alejados del deber ser de nuestra profesión. Si esto no sucede, simplemente el discurso se convierte en afirmaciones huecas, carentes de sentido y profundidad y como dice el dicho, las palabras se las lleva el viento. 


\section{La imagen como herramienta de comunicación efectiva}

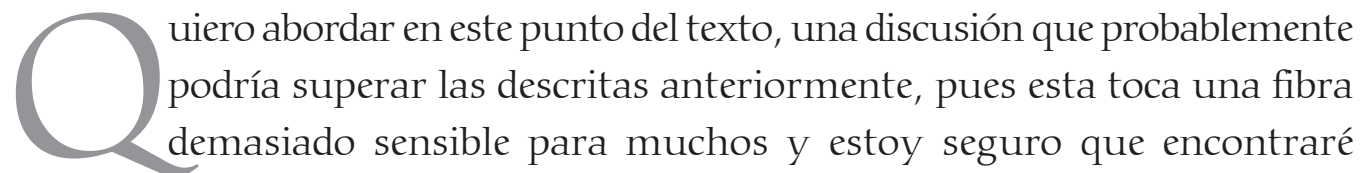
diversos detractores al exponer mis ideas; pero como el conocimiento se trata de intercambiar posiciones y posturas, aquí plasmo mi pensamiento. Veía en una de mis asignatura que cuando comenzamos a interactuar con las personas existen ciertas dimensiones que influyen a la hora de transmitir un mensaje; en primer lugar tenemos la voz. Para comunicar es indispensable contar con una voz clara, fuerte y potente que le imprima credibilidad a lo que se está diciendo. Los expertos en estos asuntos sostienen que las voces graves cuentan con una gran ventaja, frente a las voces agudas en el momento de comunicar. Además la voz de contar con el ritmo y la tonalidad adecuada para que la comunicación sea efectiva. El otro aspecto a considerar son los gestos corporales: la mirada, la manera de caminar, la forma del movimiento de las manos, entre otros gestos, son fundamentales a la hora de comunicar, puesto que dan fuerza a lo que se está diciendo y se convierte en apoyos que complementan la información que se está dando.

El otro ingrediente de esta gran receta interaccional es la imagen, y aquí sí me quiero detener un poco. Dicen los que saben que para comunicar, la imagen se constituye en el mayor elemento a tener en cuenta si se busca comunicar de manera exitosa. Cuando nos presentamos ante un grupo de personas, lo primero con lo que ellas se topan es con nuestra imagen, y se puede emitir juicios en relación al contenido del discurso por la manera en que el emisor se está mostrando al público. Un buen peinado, una vestimenta adecuada, podrían ser aspectos que marquen la manera como las personas están recibiendo nuestro mensaje. Es el impacto visual el primer contacto que tienen las personas con el profesional, no es con su voz ni mucho menos con la información que este viene a dar: es con su imagen. Por lo que es importante y no se puede dar el lujo de considerar este elemento como algo insignificante.

Me hago la pregunta, ¿con qué laboramos los trabajadores sociales? Creo que la respuesta es simple, el campo de acción de las y los trabajadores sociales, es en la sociedad, por lo tanto, la interacción con las personas se convierte en el pan de cada día para los profesionales de lo social. Si la imagen es el factor que las personas privilegian para dar validez a la información, la pregunta es si debemos o no debemos prestar atención a la imagen personal en el momento de ejercer nuestra profesión. ¿Pero qué es lo que observamos 
cuando nos damos un paseíto por las unidades académicas de trabajo social? El impacto es grande y en muchos pasillos se pueden escuchar comentarios, que las y los trabajadores sociales son los peores vestidos de la Universidad. Las excusas y hasta argumentos teóricos son infinitos: que la libre expresión, que el sistema capitalista, que este es un mundo materialista y de apariencias, en fin. Todo justifica que las compañeras asistan a clases en chores y chanclas y los compañeros en pantalonetas rotas y camisilla. iOjo! no estoy diciendo que la Universidad o el espacio laboral se debe convertir en una pasarela, tampoco estoy afirmando que se deben utilizar vestuarios ostentosos para poder tener estatus profesional, inada de eso! Lo que sostengo, es que la carrera ha utilizado argumentos vanos para justificar el descuido de la imagen, en el momento de presentarse ante la sociedad, la cual es la que valida nuestro trabajo, y nos señala como personas insignificantes por el hecho de vestir de una manera desprevenida en espacios que demandan otras características. Insisto en el hecho que no apoyo el materialismo, de hecho estoy totalmente en contra de la superficialidad que se promueve en los medios de comunicación y que se refleja hasta en la manera que nos imponen a la hora de vestir. Pero al mismo tiempo considero que las tajadas son totalmente diferentes a la sopa, es decir, el hecho de estar en contra de un sistema materialista, no es argumento para asumir nuestros espacios laborales como nuestro dormitorio o el patio de nuestra casa. Son espacios diferentes y creo que las personas a las que nosotros acompañamos merecen respeto, que se evidencia en la manera en que nosotros nos proyectamos a ellos.

Una profesora de manera muy sabia nos colocaba un ejemplo en una de sus clases, ella comentaba: "La manera en que nos vestimos denota la importancia que le damos a los espacios que asistimos. Cuando te invitan a una fiesta, un matrimonio o un grado, te preocupas por ir bien, lucir espléndido o espléndida y contar con una imagen excepcional, ipor qué? Porque ese espacio para ti es importante, tiene un valor especial. Entonces, ¿por qué de la misma manera no lo hacemos con nuestros estudios? Se supone que esto se constituye en tu proyecto de vida y por lo tanto debe tener una importancia trascendental en tu vida, y si es así, ¿por qué a la Universidad venimos como sea?". Esta es una reflexión interesante, que de una u otra forma nos muestra como la manera de vestir proyecta el interés que le damos a lo que hacemos diariamente. Pero reitero, no hablo de marcas costosas, no hablo de farándula, simplemente es una imagen que nos perfile como profesionales y que logre establecer un lazo entre la comunidad y nosotros como trabajadores sociales. 


\section{Conclusión}

ara concluir, quiero decir que amo el trabajo social, siempre le agradezco a Dios por darme la oportunidad de estudiar una carrera tan completa, que me permite introducirme en la realidad de mi entorno y me convierte en parte del cambio de las situaciones problemáticas que se viven a diario. Disfruto el trabajo social, leer, interactuar con las y los actores sociales; y partiendo de estas emociones que me genera mi profesión, observo con detalle lo que se vive en el seno de esta, y con profundo respeto y aprecio hago críticas a asuntos que desde mi visión considero pueden ser fortalecidos en trabajo social. Para perfilarnos como una profesión que impacta en la sociedad, y presentarle a las comunidades una carrera que no llena encuestas, que no regala mercados, ni mucho menos se encarga de rebajar la tarifa en un hospital. Sino por el contrario, una carrera que genera cambio, transformación, que está del lado de las y los sujetos sociales; que en su accionar acoge los derechos humanos: equidad, justicia, y desarrollo, y que es capaz por sus elementos teóricos y epistemológicos de generar empatía con las realidades sociales. Por el hecho que es capaz de introducirse en las dinámicas diarias del contexto, haciendo lecturas apropiadas que se convierten en estrategias que dan respuesta a las problemáticas más fuertes. Si. Esto es trabajo social, una herramienta poderosa en las manos de la sociedad, y por esto debe transformarse ella misma, dándose su importancia, y su valor, generando una identidad fuerte con clara proyección social y comunitaria. 

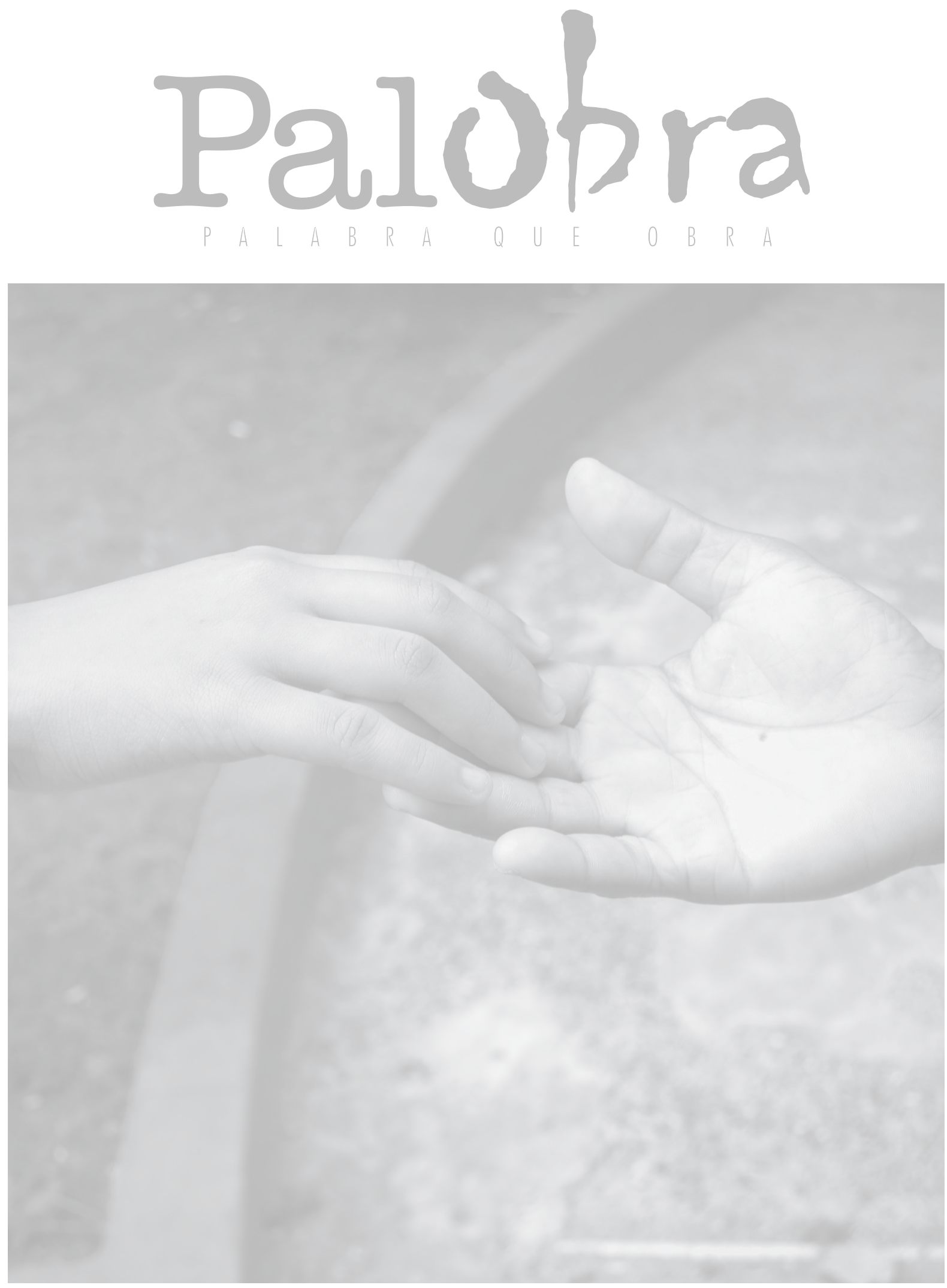